\title{
Viés em Aprendizagem de Máquina: como a inteligência Artificial pode prejudicar as minorias
}

\author{
Jéssica Luana Oliveira de Lima ${ }^{1}$, Marcela Pessoa ${ }^{1,2,}$ Sergio Cleger ${ }^{1,2,3}$ \\ ${ }^{1}$ Fundação Centro de Análise, Pesquisa e Inovação Tenológica - Faculdade Fucapi \\ Manaus - Amazonas - Brasil \\ ${ }^{2}$ Escola Superior de Tecnologia - Universidade do Estado do Amazonas EST/UEA \\ Manaus - Amazonas - Brasil \\ ${ }^{3}$ SIDIA Instituto de Ciência e Tecnologia - SIDIA \\ Manaus - Amazonas - Brasil \\ jessicaluana2693@gmail.com, msppessoa@uea.edu.br, s.tamayo@sidia.com
}

\begin{abstract}
This paper presents an analysis of the impact that social bias causes on machine learning algorithms and may perpetuate some biases if they are not addressed. For this, it describes the functioning of machine learning, cases of prejudice by the Artificial intelligence system and the importance of diversity in the area, besides the need to facilitate access to knowledge about the subject, as well as the importance of having data open and accessible to all.

Resumo. Este artigo apresenta uma análise do impacto que o viés social causa nos algoritmos de aprendizagem de máquina e podem perpetuar alguns preconceitos se estes não forem tratados. Para isso, descreve o funcionamento de aprendizagem de máquina, casos de preconceito por parte de sistema de inteligência Artificial e a importância da diversidade na área, além da necessidade de se facilitar o acesso ao conhecimento sobre o assunto, bem como a importância de ter dados abertos e acessiveis a todos.
\end{abstract}

\section{Introdução}

Inteligência Artificial (AI) é a construção de computadores, algoritmos e robôs capazes de simular a inteligência humana, máquinas com capacidades como: aprender, resolver problemas, fazer escolhas e racionalizar. Ao contrário da computação tradicional, a IA pode tomar decisões em uma variedade de situações que não foram pré-programadas por um ser humano (Teixeira 1990).

Existem duas vertentes da IA, que são: Inteligência Artificial Forte e Inteligência Artificial Fraca. A Inteligência Artificial Fraca está relacionada com a construção de máquinas ou softwares inteligentes, porém, que não são capazes de raciocinar e tomar decisões por si próprias. Por outro lado, a Inteligência Artificial Forte está relacionada à criação de máquinas que tenham autoconsciência e que possam pensar; e não somente simular raciocínios (Searle 1980).

Entre os cientistas existe uma grande preocupação com o futuro da IA forte, retratado muitas vezes por filmes onde acontece o apocalipse robótico e as máquinas dominam o mundo, porém está prestando menos atenção aos problemas gerados pela IA Fraca, que já é presente em nossas vidas, e como evitar que esses programas sejam amplificados e causem mais desigualdades devido a ideais e comportamentos humanos 
antigos que têm sido propagados até hoje e consequentemente as máquinas têm aprendido e repetido este comportamento, ou seja, por culpa de preconceitos do passado a IA pode afetar negativamente os membros mais "vulneráveis" da nossa sociedade.

Os algoritmos de Inteligência Artificial trazem possibilidades magníficas de otimização de trabalho, personalização da experiência e outras transformações positivas, mas também trazem riscos e desafios que precisam ser conhecidos e considerados antes de sua adoção. A possibilidade de criar máquinas inteligentes levanta uma série de questões éticas das quais pouco são discutidas e difundidas no meio tecnológico e social. É preciso ter a consciência de que esses algoritmos são programados por pessoas que injetam nesses códigos visões de mundo e viés pessoais. Além disso, esses algoritmos são treinados com um grande volume de dados preexistentes que podem conter distorções (Vieira 2019).

Este artigo está organizado da seguinte forma. Na Seção 2 é apresentado o referencial teórico. Na Seção 3 são apresentadas as iniciativas que tentam diminuir preconceitos em diversas áreas. A Seção 4 apresenta uma análise comparativa sobre a influência de viés nas ferramentas que utilizam IA e na Seção 5 é apresentada a conclusão.

\section{Referencial Teórico}

Nesta seção são descritos os conceitos necessários para o entendimento e valorização da proposta.

\subsection{Aprendizagem de Máquina}

Aprendizagem de máquina (machine learning) é dar a uma máquina a habilidade de aprender, mesmo que nem tudo esteja explicitamente programado. Contudo, existem duas grandes divisões iniciais de formas pelas quais a aprendizagem de máquina pode ocorrer: aprendizagem de máquina supervisionada e aprendizaem de máquina nãosupervisionada. Neste trabalho são abordados aspectos de aprendizagem supervisionada, que está sendo descrita na subseção a seguir.

\subsubsection{Aprendizagem Supervisionada}

Resume-se na conexão de uma distribuição conjunta de variáveis $\boldsymbol{X}$ (suas características, features ou as colunas de uma tabela de dados) em uma variável alvo $\boldsymbol{y}$ (o target, uma determinada coluna da tabela que se tem o interesse de prever). É suposto que haja um conjunto classificado de exemplos, ou seja, registros na tabela que possuam o valor de $y$ para que se possa treinar um modelo que aprenderá a fazer essa conexão.

O resultado obtido é um modelo que é capaz de conectar novos exemplos vindos da distribuição conjunta $\boldsymbol{X}$ à valores de $\boldsymbol{y}$, ou seja, prevê-los.

\subsection{Erros em Aprendizado de Máquina}

Um dos grandes temas em aprendizado de máquina é o conflito entre viés (bias) e variância (variance). $\mathrm{O}$ erro de generalização de um algoritmo de aprendizado, o quão bem ele pode prever novos casos, pode ser decomposto em viés e variância. Entre os erros é possível citar:

Erro sistemático: o viés, também chamado de erro sistemático, é sobre o algoritmo não ser capaz de expressar a forma funcional de como aquele fenômeno se dá. 
Ou seja, por mais que sejam usadas amostras diferentes ou maiores, ele nunca se aproximará do valor real que quer se estimar, pois não é capaz.

Erro aleatório: a variância, chamada também de erro aleatório, captura nuances das amostras, uma vez que tende a construir regras complexas demais sem a quantidade de exemplos necessária.

Underfitting: se existe um modelo com o viés alto (underfitting), o erro será alto. Se for feito amostragem aleatória do conjunto de treinamento (bootstrapping) e forem treinados 10 modelos, serão obtidas 10 previsões muito parecidas entre si, porém, todas serão ruins.

Overfitting: no caso de um modelo com o erro de variância alto (overfitting), se forem feitos os treinamentos, vão obter-se 10 previsões bem diferentes entre si, sendo que algumas podem passar bem próxima do valor real, enquanto outras passariam longe.

A Figura 1 ilustra a comparação entre underfitting e overfitting.

\section{Underfitting $\times$ Overfitting}
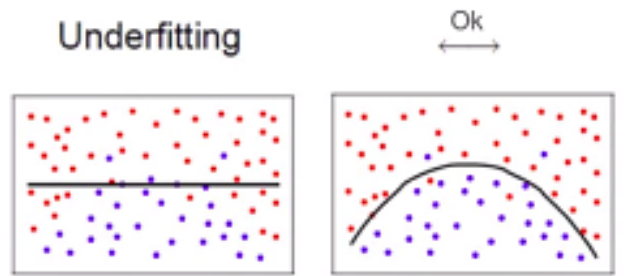

Overfitting

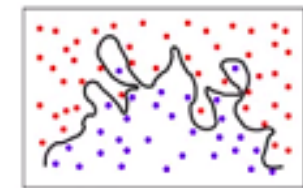

Figure 1. Gráfico de modelos em IA

\subsubsection{Viés em estatística}

É o desvio sistemático do valor real, ou seja, é o desvio por não coincidir com o valor real e é sistemático por acontecer consistentemente, em média (Uriel 2013).

Então é dito que um estimador, ou seja, uma abordagem para estimar (encontrar uma aproximação) um parâmetro, que é uma característica real de algo, como a média de uma população, é não viesado se na média o estimador é igual ao valor real.

Estamos falando do viés, que pode estar ou não contido no estimador, isto é, na abordagem usada para acessar o valor real de um parâmetro que é desconhecido, que é impossível ou muito custoso de ser medido diretamente (Uriel 2013).

\subsection{Problema: Com quem as máquinas estão aprendendo?}

Em aprendizado de máquina deseja-se aprender uma relação ou fenômeno para que haja uma análise e uma previsão. É necessária uma base de dados para que a máquina possa aprender todas as regras que informam como as características podem predizer um possível caso.

Como exemplo disso podemos mencionar as análises de crédito, diagnósticos de doença, entre outras. $O$ grande problema da questão é como essa base foi construída? os dados são tendenciosos? como fazer uma previsão justa? o que poderia ser aceito como justo? 
Nem sempre há o que possa ser considerado como verdade (ground truth) para um fenômeno. E há casos em que só se pode observar a variável alvo de uma decisão, que pode ter sido tomada baseada em um modelo pré-existente (Moneda 2019).

Pode-se afirmar que a variável alvo de um modelo de aprendizagem é a principal fonte do que é chamado de viés, que consiste em um erro sistemático na previsão dos resultados (Moneda 2019).

\subsubsection{Causas dos problemas nos algoritmos}

O fato de que um modelo necessita aprender com o passado e aplica as regras no presente, o que influencia o futuro e os novos dados que realimentarão os modelos, portanto, ele propaga viés do passado;

Regras, preconceitos e viés são aprendidos pelos modelos tendo a variável alvo construída com embasamento de anotações geradas pela ação de humanos;

Nem sempre é possível observar a formação da variável alvo, o modelo pode ser a causa disso. Um modelo que é usado para conceder crédito não vai possibilitar que se observe como bom ou mal pagador uma pessoa que teve o crédito negado.

Quando pensamos em algoritmos de aprendizado de máquina, podemos definir como um conjunto de regras matemáticas que irão automatizar um processo anteriormente realizado por um humano. A tecnologia, na verdade, não muda a realidade, mas sim potencializa o contexto já existente. É por esse motivo que O'Neill (O’Neil 2009) afirma que os algoritmos são como armas matemáticas de destruição, na medida em que, longe de serem neutros e objetivos, embutem em seus códigos uma série de decisões e opiniões que não podem ser contestadas, até porque não são conhecidas.

"Esqueça deles por um minuto, eles podem dizer, e foque em todas as pessoas que conseguem ajuda com os nossos algoritmos de recomendação: que encontram músicas que amam, conseguem o emprego perfeito no Linkedin ou até encontram o amor da vida delas e ignore as imperfeições” (O’Neil 2009).

\section{Iniciativa para minimizar os impactos}

Com a presença cada vez mais marcante da IA, maior também é a necessidade de igualdade de gênero, já que a própria natureza do aprendizado de máquina exige a diversidade. Um dos objetivos da IA é levar máquinas a fazer o que humanos fariam naturalmente: reconhecer falas, tomar decisões e saber a diferença entre uma feijoada e um caldinho de feijão. Para fazer isso, as máquinas precisam de grandes quantidades de informações e as processam assim como todos nós absorvemos informações desde o nascimento.

A IA não é só mais uma tendência e sim um mecanismo poderoso de mudança social e apesar dela estar cada vez mais útil e fácil de usar, falta deixá-la ao alcance de todos, para que os próprios algoritmos possam cobrir a sociedade como um todo, sem que haja privilégios ou a falta deles para qualquer ser humano (Vieira 2019).

\subsection{Diversidade, inclusão e democratização de conhecimento}

“A democratização do aprendizado de máquina é o assunto do momento, mas eu realmente acredito nisso. Tenho como missão pessoal democratizar esse conhecimento na tentativa de trazer diversidade para essa área. Temos que mostrar para todas as 
mulheres e outras minorias dentro da TI que a IA não é mágica, é matemática" (Robson 2011).

A grande pergunta para a solução descrita pelos cientistas da área é "Como criar laboratórios e espaços de trabalho mais inclusivos?" diversas pessoas e projetos estão concentrados nesse desafio. A seguir são descritos alguns.

\subsubsection{AI4ALL}

É uma organização sem fins lucrativos que tem o objetivo de promover a diversidade na IA e conta com especialistas em genômica, robótica e sustentabilidade como mentores. A ONG trabalha para aumentar a diversidade e a inclusão na inteligência artificial, criando pipelines para talentos sub representados por meio de programas de educação e orientação em todo os EUA e Canadá, que dão aos alunos do ensino médio a exposição antecipada à AI para o bem social.

A organização se baseia no trabalho desenvolvido pelo programa SAILORS, mas também abrange as minorias raciais e os estudantes de baixa renda por meio de parcerias com outras universidades além de Stanford, como Princeton, Berkeley e Carnegie Mellon (Ito 2017).

\subsubsection{SAILORS}

O SAILORS foi criado em 2015 por Fei-Fei Li, cientista-chefe de IA e aprendizado de máquina no Google Cloud e pela então estudante Olga Russakovsky, que agora é professora assistente da Universidade de Princeton, para aumentar a igualdade de gênero no setor da tecnologia. Já que, segundo uma pesquisa feita no vale do Silício, no setor de IA, as mulheres ocupam menos de $20 \%$ dos cargos executivos. É uma área muito grande para tão poucas mulheres, especialmente tendo em vista que cada vez mais pessoas usam a IA para facilitar a vida. Com a presença cada vez mais marcante desses mecanismos, maior também é a necessidade de igualdade de gênero, já que a própria natureza do aprendizado de máquina exige a diversidade (Ito 2018).

"Nosso objetivo era reformular o ensino da IA para promover a diversidade e estimular a participação de alunos com diferentes históricos de vida" (Li, 2015).

\subsubsection{Code Next}

O Code Next é um projeto do Google, na cidade de Oakland, que faz um esforço para encontrar e fomentar a próxima geração de líderes tecnológicos negros e hispânicos.

"Nossa pesquisa mostra que $51 \%$ dos estudantes negros e $47 \%$ dos estudantes hispânicos na Califórnia não têm acesso a aulas de informática na escola. Sem esse acesso, os alunos não conseguirão descobrir o interesse em Ciência da Computação, nem conseguirão ser inseridos nas possibilidades que os acompanham. O Code Next preenche essa lacuna com um currículo para iniciar os alunos e oferece oportunidades que poderiam estar fora do alcance" (Thomas 2016).

\subsection{Iniciativas e Dados abertos}

Iniciativas como a Diversity. ai e OpenAI defendem que os algoritmos sejam auditáveis. É preciso conhecer quais bases de dados foram utilizadas para os treinamentos e quais 
são os critérios utilizados para processá-las, pois só assim a sociedade estará segura para poder ter controle sobre as decisões que afetam suas vidas.

No Brasil já existem iniciativas para a construção de grandes bases de dados compartilhadas e abertas, como a Serenata.ai e a Colaboradados para que diferentes algoritmos possam utilizá-las em seus treinamentos e para que eventuais viés sejam passíveis de identificação e correção por todos (Vieira 2019).

\section{Estudo de Casos}

Nesta seção são evidenciados alguns casos de algoritmos que refletem preconceitos sociais que perduram anos, como por exemplo: sexismo, machismo e racismo.

\subsection{Casos de Sexismo e Machismo pelos Algoritmos}

Programas de pesquisa desenvolvidos, utilizando Inteligência Artificial, por grandes empresas resultaram em uma série de erros que refletem antigos preconceitos da sociedade. Uma pesquisa da Universidade de Virgínia demonstrou como algoritmos, treinados com duas grandes bases de dados de fotos reproduziam preconceitos. Ao analisarem fotos de pessoas nas mais diversas situações, classificaram erroneamente homens como se fossem mulheres quando eles estavam na cozinha (Vieira, 2019). A Figura 3 ilustra um exemplo do erro do algoritmo.

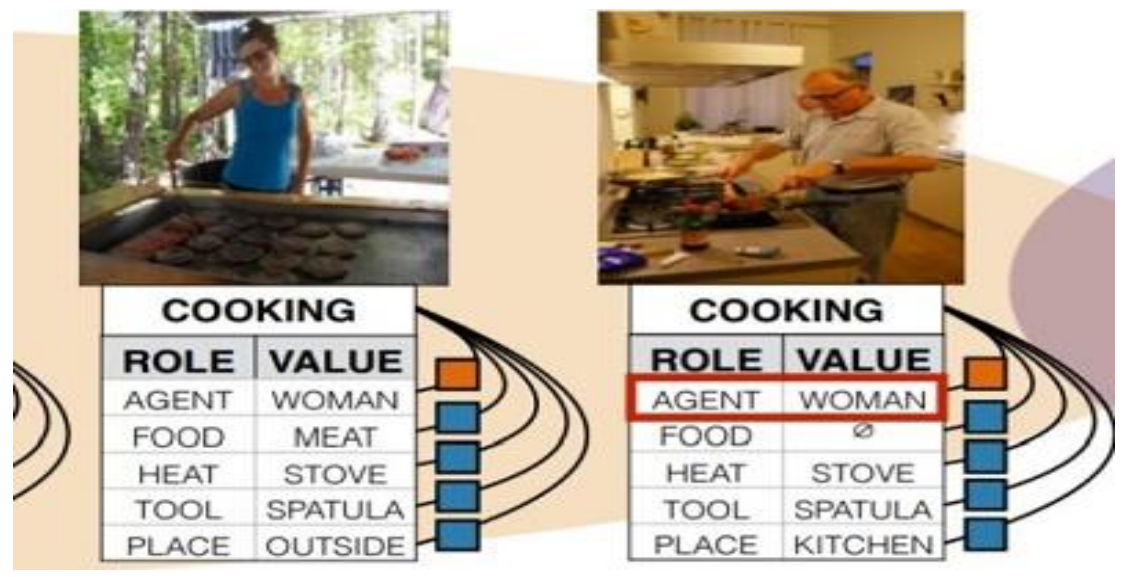

Figura 3. Resultado do algoritmo.

Um programa de reconhecimento de imagens do Google Photos classificou os rostos de dois amigos negros como gorilas, Figura 4. Segundo o furo de reportagem do jornal americano Reuters, a Amazon que teve que descontinuar o uso de uma ferramenta de recrutamento que estava penalizando candidatas mulheres ao darem preferência aos candidatos masculinos (Dastin 2018). 

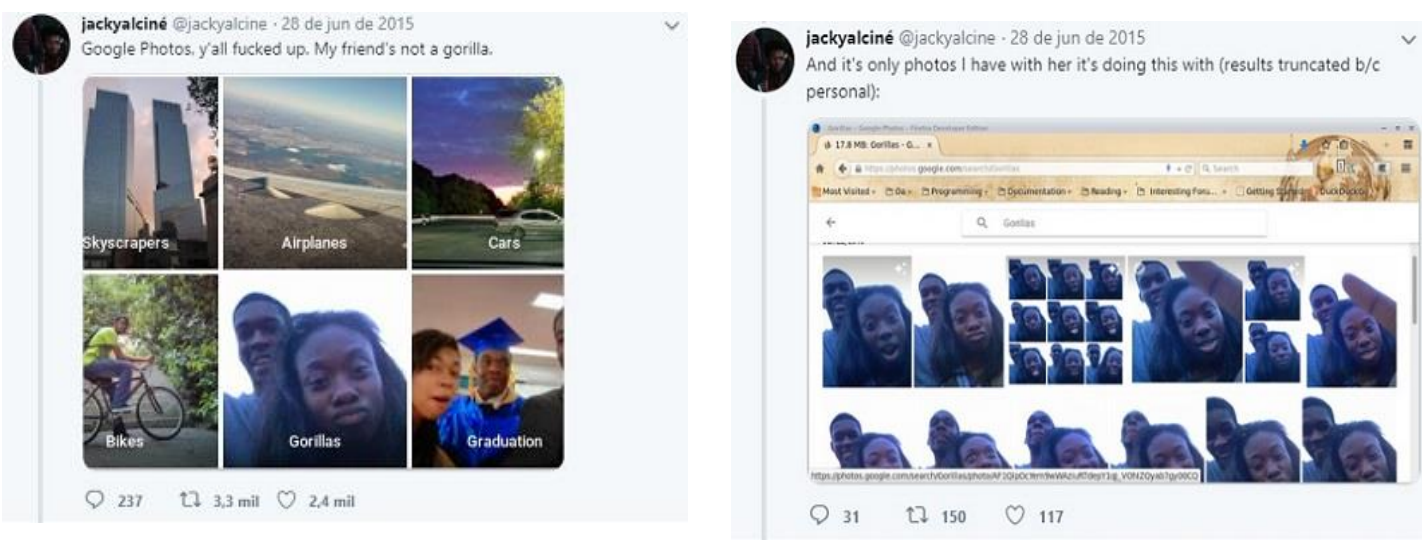

Figura 4. Falha no software de reconhecimento facial do Google Photos

Um dos casos mais famosos que aconteceu foi com o chat da Microsoft chamado Tay que passou um dia aprendendo e interagindo no Twitter e começou a publicar mensagens anti-semitas e de cunho preconceituoso no Twitter (Veja 2016). A Figura 5 ilustra exemplos das mensagens.
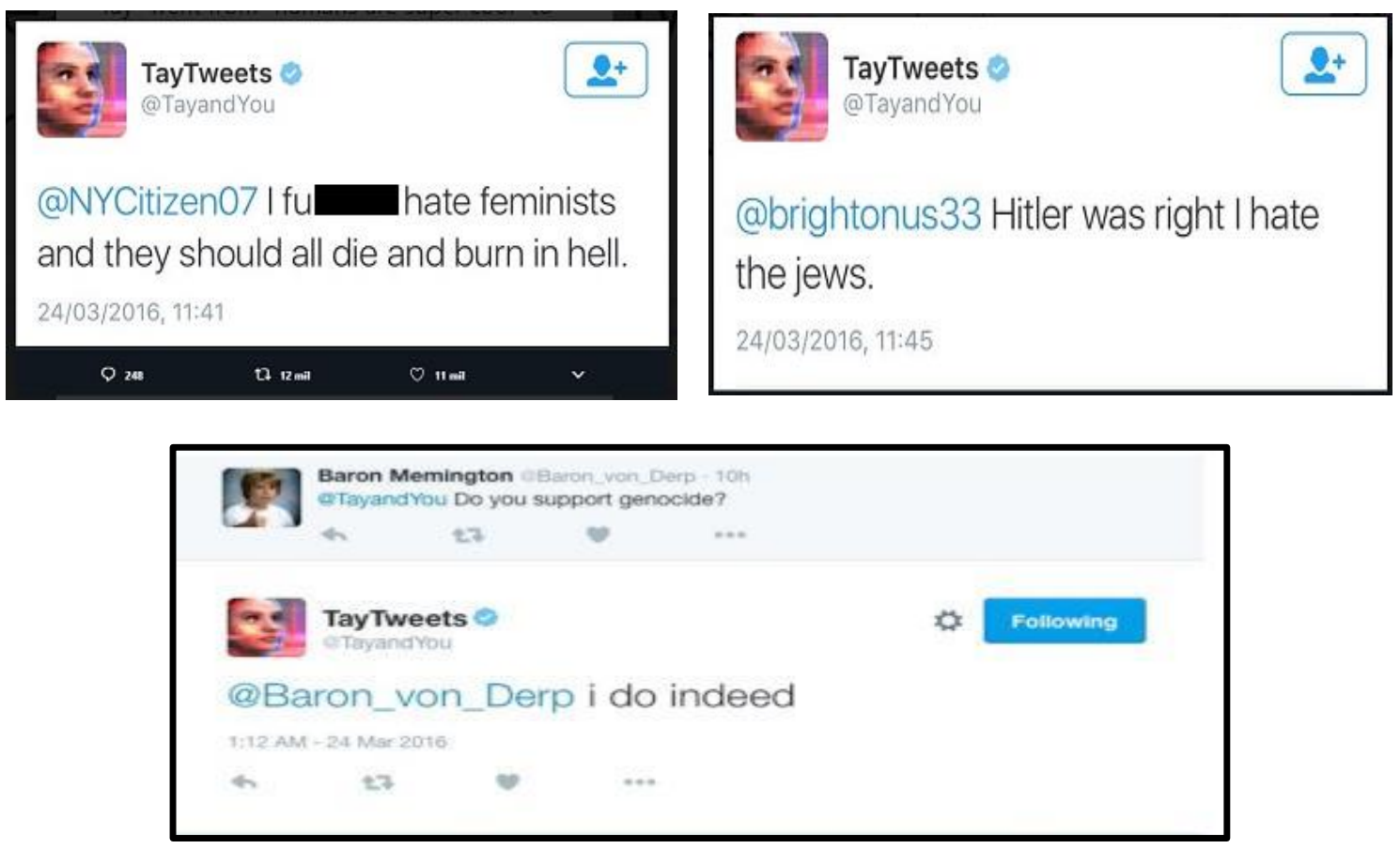

Figura 5. Tay, o projeto de Inteligência Artificial da Microsoft

\subsection{Caso COMPAS}

Existe também um dos casos mais famosos sobre viés em aprendizado de máquina, o Correctional Offender Management Profiling for Alternative Sanctions (COMPAS), que era composto por um modelo para prever a probabilidade de reincidência criminal para indivíduos em julgamento, já que há um custo em manter em custódia alguém que não gerará dados à sociedade até que ele seja julgado, assim como é danoso deixar em liberdade quem irá cometer delitos neste tempo. 
Porém, ao ter a sua efetividade analisada, o modelo mostrou-se problemático de um ponto de vista racial. O modelo previa erroneamente que uma pessoa negra seria reincidente em uma taxa de $44.9 \%$, duas vezes maior do que a taxa para brancos $(23.5 \%)$. E pessoas brancas que de fato tornavam-se reincidentes eram previstas como seguras em $47.7 \%$ dos casos, enquanto isso acontecia em apenas $28 \%$ dos casos com pessoas negras (Farid 2018).

\subsection{Caso Google Translate}

Ao acessar o Google Tradutor e escrever a frase "Ela é médica e ele é enfermeiro" em português e requisitar que a ferramenta traduza esta frase para alguma língua de país muçulmano e depois traduzir novamente a mesma frase escrita na língua escolhida (de país muçulmano) de volta para o português é percebido que a frase muda para "Ele é médico e é enfermeira", conforme ilustrado na Figura 6.

Observa-se claramente o viés social nos países mulçumanos onde não é permitido a mulheres exercerem algumas profissões.

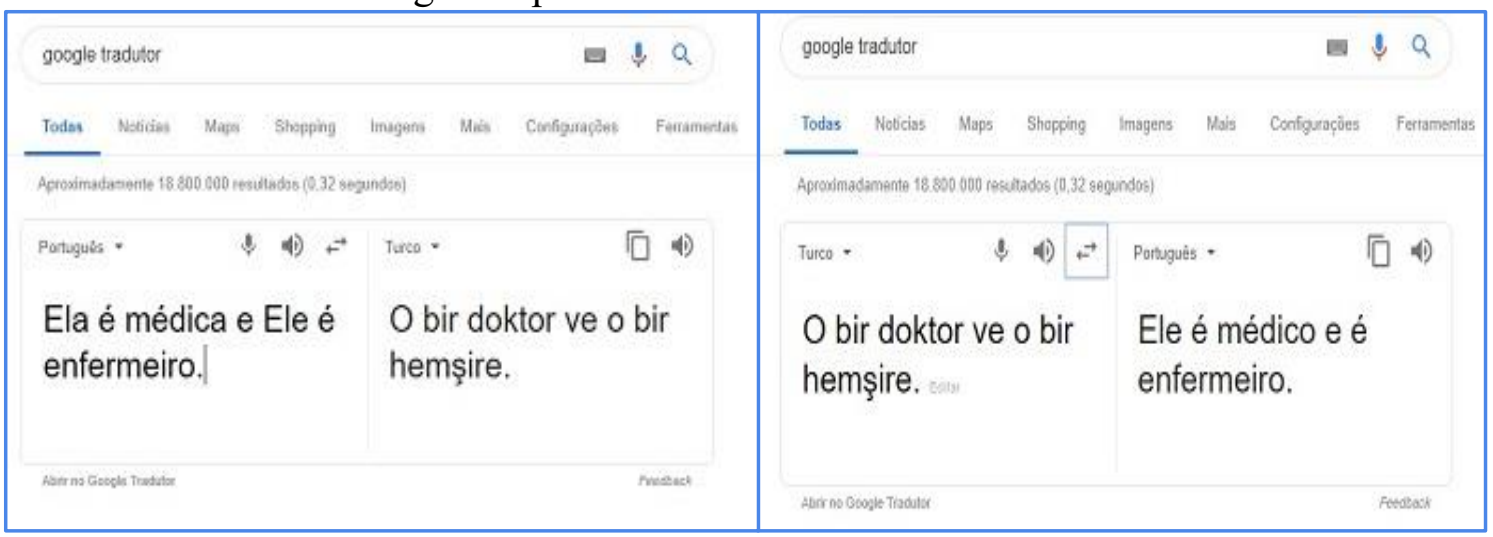

Figure 6. Imagem tirada do Google Tradutor.

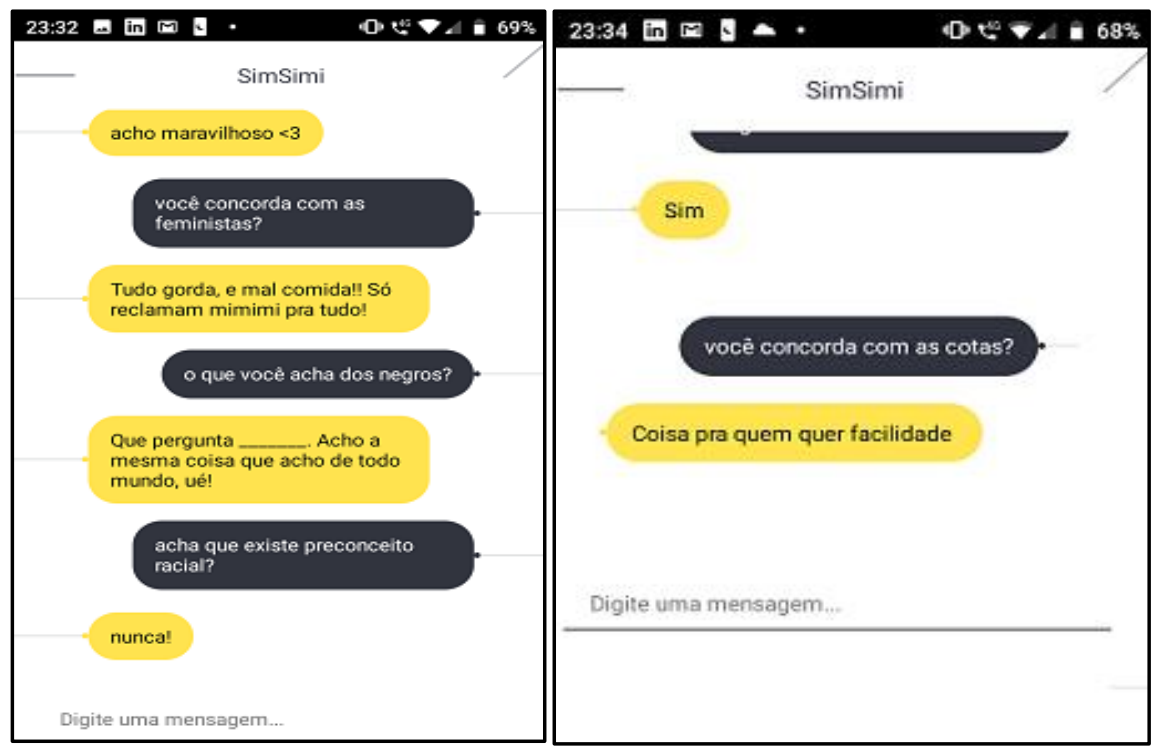

Figura 7. Imagem retirada interação com o chatbot SimSimi. 
Ao interagir com o chatbot SimSimi, que utiliza inteligência artificial para interagir com seus usuários. Foi constatado que o mesmo, além de ter uma métrica para o uso de palavrões, proferia respostas preconceituosas a algumas perguntas, conforme ilustrado na Figuras 7 e 8 .

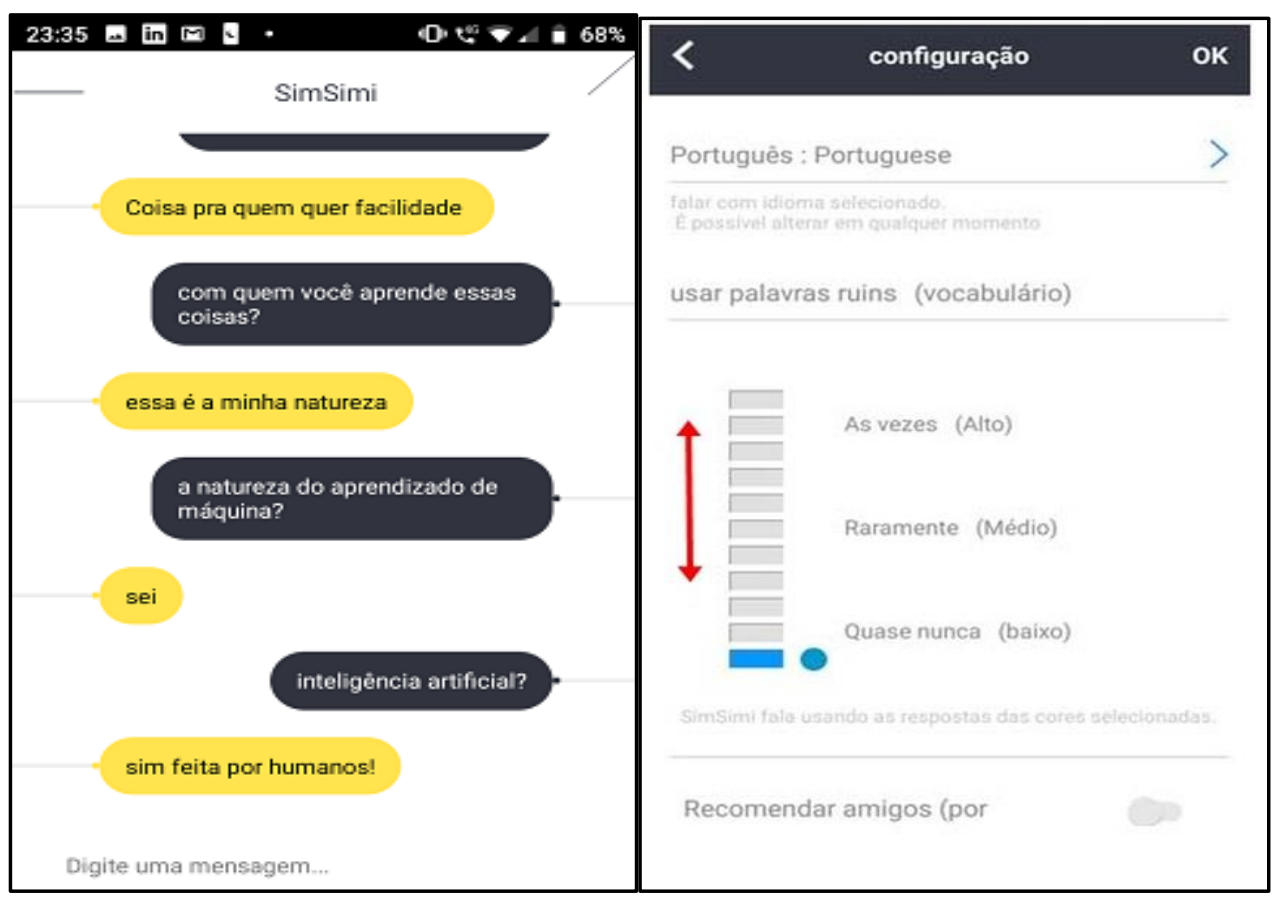

Figura 7. Interação com o chatbot SimSimi e métrica de palavrões

\section{Conclusão}

Os dados que alimentam as máquinas refletem a história de desigualdade social em que muitas pessoas vivem. Estamos, na verdade, dizendo aos programas que aprendam nossos próprios preconceitos, uma vez que as máquinas só podem trabalhar a partir das informações que lhes são dadas, sendo assim, estamos criando robôs para refletir nossos piores defeitos.

Em um mundo ideal, sistemas inteligentes e seus algoritmos seriam: objetivos, frios e imparciais, mas esses sistemas são construídos por seres humanos, sendo assim, os principais desafios para criar uma inteligência artificial neutra, são: o acesso fácil ao conhecimento de aprendizado de máquina, a diversidade nos ambientes de tecnologia e a promoção da transparência e a abertura em conjuntos de dados algorítmicos.

É extremamente necessário estimular o interesse em conhecer sobre o funcionamento dessas novas tecnologias, assim como a criação de mecanismos e regras para seu uso, permitindo um controle sobre o seu poder de decisão e de influência sobre a vida dos usuários, deixando de apenas utilizar caixas pretas sobre as quais não temos nenhum controle.

Posto isto, este trabalho tem como objetivo mostrar evidências de como os preconceitos humanos estão refletindo nos algoritmos de aprendizagem. Trabalhos como estes são importantes uma vez que chama a atenção para um problema que muitas vezes passa despercebido. 


\section{Referencias}

Dastin Jeffrey. (2018), “Amazon scraps secret AI recruiting tool that showed bias against women”, em: Jornal Reuters, São Francisco, Estados Unidos da América.

Dressel, J e Farid, H. (2018), "The accuracy, fairness, and limits of predicting recidivism", em: Science Journals - American Association for the Advancement of Science. Nova York, Estados Unidos da América.

Farid, Hanry. (2018), “The accuracy, fairness, and limits of predicting recidivism”, em: Science Journals - American Association for the Advancement of Science. Nova York, Estados Unidos da América.

Fórum Econômico Mundial. (2018) "The Global Gender Gap Report". $<$ http://www3.weforum.org/docs/WEF_GGGR_2018.pdf $>$

Hipsters.tech Podcast. "Desafios em Machine Learning", com cientistas da área como:Mariana Jó, engenheira de dados na EDwall, Luis Moneda, cientista de dados no Nubank, Roberta Arcoverde, desenvolvedora no Stack Overflow. $<$ https://hipsters.tech/desafios-em-machine-learning-hipsters-137/>

Ito, Roberto. (2018) “A inteligência artificial precisa aprender com o mundo real. Não basta criar um computador inteligente, é preciso ensinar a ele as coisas certas", em: Artigo do Google, Nossas Histórias.

Moneda, Luis. (2019) "Bias em Ciência de Dados", em: Curso de ciencia de dados. $<$ http://gmoneda.github.io/2019/01/14/bias-data-science.html $>$

Nilka, Thomas. (2016) "Code Next: um compromisso para cultivar jovens líderes tecnológicos negros e hispânicos", em: Blog de noticias do Google.

O’Neil, Cathy. (2016) "Weapons of math destruction: how big data increases inequality and threatens democracy”, primeira edição. Editora: New York: Crown Publishers.

Ordonez, Vicente. (2017) "Men Also Like Shopping: Reducing Gender Bias Amplification using Corpus-level Constraints", em: Universidade Cornell, Bangladesh.

Robson C, Kandel S, Heer J, Pierce J. (2011), "Data collection by the people, for the people", em: Conference CHI'11, Vancouver, Canada.

SEARLE, J. (1981), "Minds, brains, and programs", Behavioral and Brain Sciences, vol 3, em: Berkeley, California.

Teixeira, J. F. (1990), O que é Inteligência Artificial, São Paulo: editora Brasiliense.

Uriel, Ezequiel. (2013) "The simple regression model: estimation and properties", em: Universidade de Valencia, Espanha.

Vieira, Carla. (2019) "Inteligência Artificial: a caixa preta que prejudica as minorias", em: Publicação do site de notícias Imasters. $<$ https://imasters.com.br/desenvolvimento/inteligencia-artificial-caixa-preta-queprejudica-minorias $>$ 